\title{
Linfomas asociados a infección por VIH en pacientes del Hospital Clínico San Borja Arriarán / Fundación Arriarán 2001-2008
}

\author{
Álvaro Pizarro, Hernán García, Ana Riquelme, Javiera Carmona y Claudia Cortés
}

\section{Lymphomas associated with HIV infection in patients at the Hospital San Borja Arriarán /Fundación Arriarán 2001-2008, Santiago, Chile}

Introduction: Hodgkin lymphomas (HL) and non Hodgkin lymphomas (NHL) are frequently associated to acquired immunodeficiency syndrome in adults. Objective: To systematize the clinical features and histological characteristics of lymphomas in AIDS patients, its treatment and outcomes in our institution. Patients and Methods: Retrospective analysis of patients with HIV-associated lymphoma between January 2001 and December 2008 at the San Borja Arriarán Hospital complex. Results: Information was obtained from 30 patients with NHL and 7 with HL, with a median of 40 years. The majority of tumors were Burkitt lymphoma (47\%), diffuse large cell lymphoma B-cell (37\%) and NHL of T lineage (10\%). There was no CNS or cavities lymphoma. Almost all patients $(86.7 \%)$ with NHL were treated with CHOP chemotherapy, $57 \%$ of those receiving treatment had progression or relapse from complete remission. A rescue chemotherapy was indicated in 4 patients. $73 \%$ of patients receiving CHOP, complete 5 to 6 cycles of chemotherapy. The use of CHOP chemotherapy for the subgroup of patients with Burkitt lymphoma achieved low rates of complete remission and frequent relapse and disease progression, showing that CHOP was ineffective in improving survival, especially in high risk patients. We found statistically significant differences in survival according to IPIae (International prognostic Index age-adjusted). Conclusion: Non-Hodgkin lymphoma in HIV patients treated with chemotherapy protocols PANDA persists in our environment as a disease with a poor prognosis compared with findings in the international literature. The incorporation of new drugs of proven utility as rituximab and specific schemes chemotherapy could improve these results. The establishment of prognostic groups established by IPIae can guide clinical work for the use of chemotherapy tailored to their specific risk and optimized according to histological type.

Key words: HIV/AIDS, HIV associated lymphoma, Burkitt lymphoma, international prognostic index (IPI)

Palabras clave: VIH/SIDA, linfoma asociado a VIH, linfoma de Burkitt.

\section{Introducción}

$\mathrm{E}$ 1 linfoma no Hodgkin (LNH) es una de las neoplasias más frecuentes observadas en pacientes con infección por virus de inmunodeficiencia humana $(\mathrm{VIH})^{1}$. El LNH y el linfoma de Hodgkin (LH) están asociados a la infección por VIH desde los inicios de la epidemia, siendo el primero considerado una enfermedad definitoria de SIDA.

Se ha estimado que la incidencia de LNH en pacientes con infección por VIH es sobre 100 veces la encontrada en la población general ${ }^{2}$ y en los casos de LH es entre 5 y 15 veces la tasa de la población general ${ }^{3}$. En los pacientes con infección por VIH, el linfoma difuso de células grandes estirpe B (LDCGB) y linfoma de Burkitt (LB) son las formas más comunes de LNH. En el caso de los LH, las variantes histológicas de celularidad mixta y depleción linfocitaria son las más frecuentes ${ }^{4}$. Ciertos tipos de linfoma ocurren específicamente en pacientes infectados con VIH, como el linfoma primario de cavidades (PEL, por su sigla en inglés -primary effusion lymphoma) y linfoma plasmoblástico. En pacientes con infección por VIH ocurre más frecuentemente la presentación extranodal en el sistema nervioso central (SNC) de linfoma difuso de células grandes (LDCG) así como el linfoma primario de $\mathrm{SNC}^{5}$.

El uso de terapia anti-retroviral (TARV) altamente efectiva desde 1996, ha provocado cambios epidemiológicos en los pacientes. La cohorte EuroSIDA reportó un incremento relativo de LNH desde 4\% en 1994 a $16 \%$ en 1998 en la proporción de neoplasias diagnosticadas en esta población, debido a la disminución del sarcoma de Kaposi ${ }^{6}$. Por otra parte, un meta-análisis de 23 estudios de cohortes encontró una disminución de linfomas relacionados a SIDA de 0,62\% por año entre 1992-1996 a $0,36 \%$ por año entre 1997-1999. La disminución ocurrió especialmente en linfoma primario de SNC y linfoma inmunoblástico, asociados a recuentos de linfocitos
Hospital San Borja Arriarán, Santiago, Chile. Servicio Hematología (AP, HG, AR). Universidad de Chile, Santiago, Chile.

Facultad de Medicina Interna de Medicina (JC). Fundación Arriarán (CC).

Trabajo realizado en el complejo Hospitalario San Borja ArriaránFundación Arriarán. Los autores declaran no tener conflictos de interés. Proyecto sin financiamiento específico.

Recibido: 11 de abril de 2012 Aceptado: 22 de noviembre de 2012

Correspondencia a: Álvaro Pizarro Q. valopizarro@hotmail.com 
CD4 bajo 50 céls $/ \mathrm{mm}^{3}$, situación que no ocurre con el linfoma de Burkitt que se presenta con valores mayores de linfocitos CD4?.

El beneficio de TARV combinada con quimioterapia en forma simultánea, ya sea en dosis plenas o reducidas, ha mostrado su eficacia y tolerancia permitiendo una opción terapéutica con fines curativos en pacientes con LNH asociados a $\mathrm{VIH}^{8}$.

El uso de TARV en Chile, primero con biterapia desde el año 1997 y triterapia a partir del año 2003, ha modificado la situación epidemiológica de los pacientes con infección por VIH, evidenciando una progresiva disminución de la tasa de mortalidad que alcanzó su máximo en el 2001 con 3,6 por 100.000 habitantes, iniciando un lento descenso, llegando en el año 2007 a 2,4 por 100.000 habitantes ${ }^{9}$.

En Chile, Wolff y cols., publicaron una serie de 486 casos de pacientes infectados por VIH, entre los años 1988 y 1993 describiendo cuatro casos de LNH con una incidencia de $0,8 \%$. Una publicación posterior del mismo grupo con 316 pacientes, entre 1996 y 1999, reportó cuatro casos de LNH con una incidencia de $1,3 \%{ }^{10,11}$.

En Chile, según datos de la Comisión Nacional del SIDA (CONASIDA), a mayo de 2004, la incidencia acumulada era de 26 casos en 3.218 pacientes y la prevalencia de $0,8 \%{ }^{12}$.

Un reporte de 14 casos (10 LNH y 4 LH) del Hospital Barros Luco Trudeau entre 1990-2002 mostró una incidencia de $0,68 \% \mathrm{y}$, a pesar de tratamiento quimioterápico con CHOP (ciclofosfamida, doxorubicina, vincristina, prednisona) y ABVD (adriamicina, bleomicina, vinblastina, dacarbazina) respectivamente, presentaron una mortalidad global de $71 \%$, por progresión tumoral y/o sepsis $^{13}$.

Se diseñó el presente estudio con el objetivo de determinar las características clínicas e histológicas de los pacientes con infección por VIH con linfoma, atendidos entre el año 2001 y 2008 en el Hospital San Borja Arriarán, la terapia recibida, su respuesta a tratamiento, evaluación de factores pronósticos y sobrevida global.

\section{Pacientes y Método}

\section{Pacientes}

Ingresaron al estudio los pacientes con diagnóstico de linfoma e infección por VIH realizado entre los años 2001 y 2008, identificados según el registro estadístico de pacientes con esta patología y biopsias de médula ósea en el Departamento de Hematología del Hospital San Borja Arriarán y la base de datos electrónica de los pacientes controlados en Fundación Arriarán, centro de atención de enfermedad por VIH correspondiente al Servicio de Salud Metropolitano Central. Se identificaron las características demográficas, variables en relación a su síndrome linfo- proliferativo como forma de presentación e histología, sitio principal de presentación clínica, presencia de masa voluminosa, estadio clínico según clasificación de Ann Arbor ${ }^{14}$ y la presencia de síntomas constitucionales. En relación al tratamiento de su enfermedad neoplásica se registró: tipo de quimioterapia inicial y de rescate, asociación con radioterapia y número de ciclos y respuesta al tratamiento post finalización de la quimioterapia según criterios revisados de respuesta para linfoma ${ }^{15}$. Para evaluar el pronóstico se aplicó el índice internacional pronóstico ajustado a edad (IPI ae) ${ }^{16}$ para los LNH y el puntaje de Hasenclever ${ }^{17}$ para los casos de LH avanzados. En relación con su infección por VIH se estableció la fecha de diagnóstico de su patología infecciosa y neoplásica, recuento de linfocitos CD4 al diagnóstico de la neoplasia y si estaban recibiendo TARV. Finalmente se estableció la sobrevida global de los pacientes, determinada desde el momento del diagnóstico de la neoplasia.

\section{Definiciones}

Para establecer el estadio de los pacientes se utilizó la clasificación de Ann Arbor $^{14}$. Se definió masa voluminosa como una masa $\geq 7 \mathrm{~cm}$ de diámetro mayor y los síntomas constitucionales catalogados con la letra B en caso de fiebre mayor de 38 grados Celsius, baja de peso mayor de $10 \%$ del peso corporal en los seis meses previos al diagnóstico y sudoración nocturna y A en caso de ausencia de todos los síntomas. Se utilizó con fines pronósticos IPI (ae) para los LNH asignándole un punto en caso de deshidrogenasa láctica (LDH) elevada, etapificación avanzada (estadios III y IV) y escala de estado funcional $\geq 2$. Se utilizó la siguiente escala de estado funcional: 0 para completamente activo, 1 restringido de actividades extenuantes, 2 ambulatorio pero restringido con más de $50 \%$ del tiempo en pie, 3 actividad muy restringida y en cama más de $50 \%$ del tiempo, 4 completamente incapacitado, en cama o silla permanente. Se utilizó IPI (ae) en vez de IPI por su fácil aplicación y su creciente uso en la literatura médica relacionada. No se utilizó RIPI (índice internacional pronóstico revisado $)^{18}$ debido a que en el tratamiento no se utilizó rituximab en caso alguno. Se utilizó para los LH avanzados el puntaje de Hasenclever ${ }^{17}$, asignándole 1 punto en caso de la presencia de algunos de los siguientes factores: edad $\geq 45$ años, sexo masculino, estadio IV, albúmina $<$ de $4 \mathrm{~g} / \mathrm{dl}$, hemoglobinemia $<10,5$ $\mathrm{g} / \mathrm{dl}$, recuento leucocitario $>15.000 / \mathrm{mm}^{3}$, linfocitario $<$ de 600 céls $/ \mathrm{mm}^{3}$ o $<8 \%$ total de leucocitos. En el caso de LH localizado se han descrito diversos índices pronósticos que incluyen tamaño de la masa mediastínica, número de sitios nodales comprometidos y velocidad de sedimentación eritrocitaria ${ }^{19}$; sin embargo, también se ha ocupado el índice de Hasenclever. En nuestra casuística sólo hubo un caso de LH localizado por lo que se decidió mantener el mismo índice pronóstico ${ }^{17}$. 


\section{Tratamiento}

Se aplicaron los protocolos sugeridos por el Programa Nacional de Cáncer del Adulto (PANDA) ${ }^{20}$. En nuestra casuística se utilizó para LNH esquemas $\mathrm{CHOP}$ en dosis plenas, $\mathrm{CHOP}$ en dosis reducidas en $50 \%$ para ciclofosfamida y doxorubicina; el resto de la quimioterapia sin cambios. En relación a las quimioterapias de rescate, se utilizó esquema ESHAP. (metilprednisolona, etoposido, cisplatino, citarabina) y MINE (mitoxantrona, etoposido, ifosfamida, mesna) y quimioterapia específica para linfoma de Burkitt.

\section{Criterios de respuesta}

Se aplicaron criterios según protocolos PAN$\mathrm{DA}^{20}$. Remisión completa: normalización de todos los signos clínicos, radiológicos y bioquímicos durante al menos tres meses. Cuando hay compromiso visceral, las nuevas biopsias deben ser negativas. Los ganglios comprometidos deben regresar al tamaño normal o deben ser $<1,5 \mathrm{~cm}$ en su diámetro transverso mayor, para ganglios de más de $1,5 \mathrm{~cm}$ antes del tratamiento. Ganglios comprometidos entre 1,1 y $1,5 \mathrm{~cm}$ de diámetro deben reducirse a menos de $1 \mathrm{~cm}$. Remisión parcial: reducción $>50 \%$ del tamaño de los tumores accesibles a la palpación y/o radiología, con mejoría clínica y de parámetros de laboratorio. Enfermedad estable: falla para alcanzar remisión completa o remisión parcial. Recaída o enfermedad progresiva: Cualquier lesión nueva o que aumenta $>50 \%$ en sitios previamente comprometidos.

Las biopsias fueron analizadas en la unidad de Anatomía Patológica del Hospital San Borja Arriarán y centro de referencia correspondiente para estudio inmuno- histoquímico (Instituto de Anatomía Patológica Ltda).

\section{Análisis estadístico}

Los datos continuos fueron analizados en medianas y desviación estándar. Los datos categóricos se analizaron como números absolutos y porcentajes. Se estableció la sobrevida global de los pacientes, determinada desde el momento del diagnóstico de la neoplasia. Se realizaron curvas de Kaplan Meyer diferenciadas por IPI y por tipo histológico.

\section{Resultados}

Ingresaron al estudio 37 pacientes. Treinta pacientes correspondían a LNH y 7 pacientes a LH. Sus características generales están descritas en la Tabla 1.

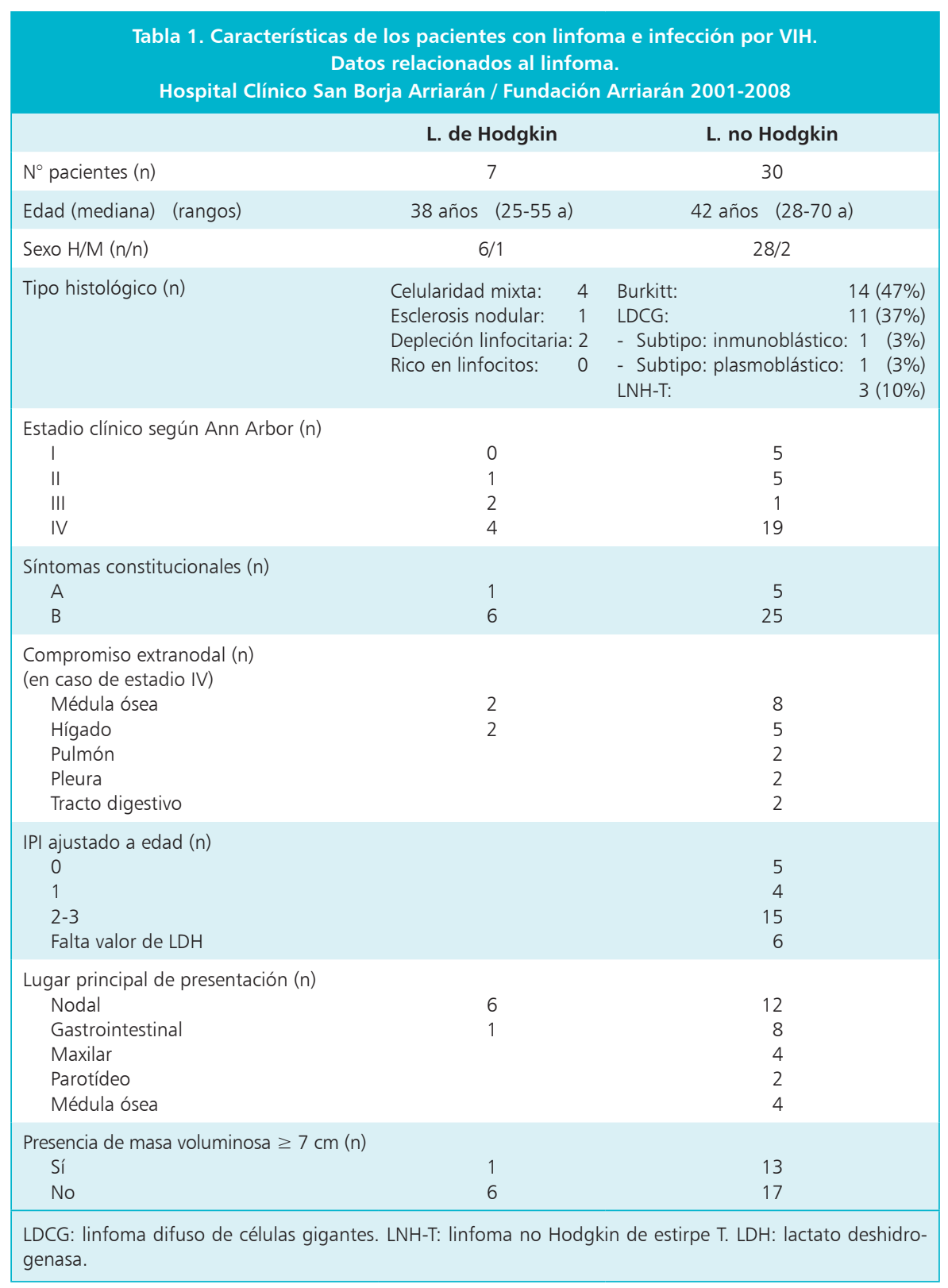

La mediana de edad fue de 40 años para todos los pacientes (rango intercuartil: 34-49 años), 38 años para pacientes con LH y 42 años para pacientes con LNH. La distribución por género fue: masculino $93 \%$ en casos de LNH y $86 \%$ en LH.

Los tipos histológicos predominantes correspondieron a linfoma de Burkitt en 47\% de los casos (14/30), LDCGB en $37 \%$ (11/30), y LNH de estirpe T con 10\% (3/30). Otros tipos histológicos correspondieron a casos aislados de linfoma plasmoblástico e inmunoblástico. No se diagnosticaron LNH del SNC ni linfoma de cavidades.

La mayoría de los pacientes de la serie presentó un estadio avanzado de la enfermedad neoplásica (20 casos para LNH 
y 6 casos para LH) que corresponden a estadio III y IV de la clasificación de Ann Arbor y con la presencia de síntomas B en 25 (83,3\%) casos de LNH y 6 casos $(85,7 \%)$ de LH. En la Tabla 1 se describen datos adicionales. En $37,8 \%$ de los pacientes se diagnósticó la infección por VIH durante el proceso diagnóstico de su enfermedad linfoproliferativa, la mediana del recuento de linfocitos CD4 basal fue de 162 céls $/ \mathrm{mm}^{3}$ con un rango intercuartil $38-235$ y se constató que casi la totalidad de los pacientes (36/37) recibió TARV altamente efectiva, con al menos tres fármacos (Tabla 2).

El tipo de tratamiento del LNH se presenta en la Tabla 3. La quimioterapia inicial ofrecida para los casos de LNH consistió en un esquema CHOP con dosis total en 21 pacientes $(70 \%)$ y con dosis reducida en cinco pacientes $(16,6 \%)$ : a un paciente se le ofreció quimioterapia citoreductora con ciclofosfamida y prednisona con posterior progresión de su enfermedad y muerte, un paciente recibió sólo radioterapia por ser estadio localizado I-A y dos pacientes no recibieron quimioterapia por progresión de la enfermedad tumoral y sepsis con fallecimiento posterior, uno de ellos por presentar sepsis con foco abdominal -una probable colangitis-y otro por shock hipovolémico post resección intestinal.

De los 26 pacientes con LNH que se les ofreció CHOP como esquema inicial, 15 (57\%) presentaron progresión de su enfermedad o recaída desde la remisión completa, ofreciéndoles una quimioterapia de rescate a cuatro pacientes (tres con linfoma de Burkitt y uno con LDCGB). En el caso de LDCGB se indicó esquema con ESHAP, no logrando remisión completa y falleciendo al mes de inicio de quimioterapia de rescate, por progresión de la enfermedad.

Los pacientes con linfoma de Burkitt recibieron en su mayoría (13/14) CHOP como terapia inicial, tres pacientes recibieron quimioterapia de rescate luego de recaer. En dos se indicó quimioterapia específica para este tipo de linfomas, uno de ellos recibió prefase que incluye prednisona, ciclofosfamida, mesna y un ciclo block AA que incluye dexametasona, ifosfamida, mesna,

Tabla 2. Características de los pacientes con linfoma e infección por VIH. Datos relacionados a la infección por VIH.

Hospital Clínico San Borja Arriarán / Fundación Arriarán 2001-2008

\begin{tabular}{|lcc|} 
& L. de Hodgkin & L. no Hodgkin \\
Diagnóstico de infección por VIH & & \\
$\quad$ Previo al dg linfoma ( $n)$ & 3 & 10 \\
$\quad$ Durante dg linfoma (n) & 4 & $151(43-231)$ \\
Nivel de CD4 al diagnóstico del linfoma & $228(76-442)$ & $29(96,6 \%)$ \\
Mediana (rango intercuartil) & $7(100 \%)$ & \\
Triterapia durante quimioterapia (n) & 4 & \\
Triterapia previa a la QT & 3 & \\
Triterapia post inicio de QT & & \\
\hline
\end{tabular}

vincristina, etoposido y citarabina y otro paciente prefase y un block AA y un block BB que incluye dexametasona, ciclofosfamida, mesna, vincristina y doxorubicina. Los tres pacientes fallecieron por progresión de su enfermedad tumoral, no alcanzando a completar tratamiento en ninguno de los casos.

El 73\% de los pacientes que recibió CHOP logró completar 5 a 6 ciclos de quimioterapia.

En el caso de los pacientes con $\mathrm{LH}$, todos recibieron quimioterapia ABVD (doxorubicina, bleomicina, vinblastina, dacarbacina), completando 6/7 (86\%) la totalidad de los ciclos indicados. Tres pacientes presentaron progresión o recaída de enfermedad.

Se evaluó la respuesta al tratamiento considerando los factores que establecen pronóstico como el tipo histológico, estadio y el IPIae en los casos de LNH (Tabla 4) y el puntaje de Hasenclever en los LH. El 63,6\% de los pacientes con LDCG logró remisión completa y $35,7 \%$ de los linfomas de Burkitt; los tres casos de linfoma de estirpe $\mathrm{T}$ progresaron. El $68 \%$ de los pacientes con estadio IV y $76,9 \%$ de los pacientes con estadio según IPI ae 2-3 (alto riesgo) presentaron progresión de enfermedad.

Tres de cuatro con LH en estadio IV presentaron progresión o recaída de enfermedad.

El análisis de sobrevida global y según tipo histológico e IPIae se observa en la Figura 1. Se encontró diferencia estadísticamente significativa en sobrevida según el valor del IPIae, no lográndose establecer diferencia según tipo histológicos entre LDCGB y linfoma de Burkitt (Figura 2).

Tabla 3. Tratamiento del linfoma no Hodgkin en pacientes con infección por VIH. Hospital Clínico San Borja Arriarán / Fundación Arriarán 2001-2008

$\begin{array}{lrr}\text { Terapia } & \mathbf{n} & \mathbf{( \% )} \\ \text { Quimioterapia cito-reductora } & 1 & (3,3 \%) \\ \text { Quimioterapia CHOP en dosis total } & 18 & (60 \%) \\ \text { Quimioterapia CHOP en dosis total + radioterapia } & 3 & (10 \%) \\ \text { Quimioterapia CHOP en dosis reducida } & 5 & (16,7 \%) \\ \text { Radioterapia } & 1 & (3,3 \%) \\ \text { Sin tratamiento } & 2 & (6,7 \%) \\ \text { Tipo de tratamiento recibido de rescate: } & 15 & \\ \text { Sin tratamiento } & 8(53,3 \%) \\ \text { QT ESHAP } & 1 & (6,7 \%) \\ \text { QT Burkitt } & 2 & (13,3 \%) \\ \text { Radioterapia paliativa } & 3 & (20 \%) \\ \text { ESHAP-MINE } & 1 & (6,7 \%) \\ \text { Linfoma de Burkitt } & 14 \\ \text { QT CHOP } & 8(57,1 \%) \\ \text { QT CHOP + radioterpia } & 2 \quad(14,3 \%) \\ \text { QT CHOP + QT Burkitt } & 2 \quad(14,3 \%) \\ \text { QT CHOP + ESHAP-MINE } & 1 & (7,1 \%) \\ \text { Sin tratamiento } & 1 & (7,1 \%) \\ \text { CHOP (ciclofosfamida, doxorubicina, vincristina, } & \text { prednisona); } \\ \text { ESHAP (metilprednisolona, etoposido, cisplatino } & \text { y citarabina); } \\ \text { MINE (mitoxantrona, etopósido, ifosfamida, mesna). } & \\ & \end{array}$




\section{Tabla 4. Respuesta a tratamiento de linfomas no Hodgkin en pacientes con infección por VIH. Hospital Clínico \\ San Borja Arriarán / Fundación Arriarán 2001-2008}

\begin{tabular}{lrccc|} 
& $\mathbf{n}$ & $\begin{array}{c}\mathbf{R C} \\
\mathbf{n}(\%)\end{array}$ & $\begin{array}{c}\mathbf{R P} \\
\mathbf{n}\end{array}$ & $\begin{array}{c}\text { Enfermedad } \\
\text { progresiva } \\
\text { (n) }\end{array}$ \\
Tipo histológico & & & & \\
$\quad$ LDCG & 11 & $7(63,6 \%)$ & & 4 \\
$\quad$ Burkitt & 14 & $5(35,7 \%)$ & 2 & 7 \\
LNH-T & 3 & & & 3 \\
Plasmoblástico & 1 & $1(100 \%)$ & & \\
Inmunoblástico & 1 & & & 1 \\
Estadio clínico & & & & \\
I & 5 & $5(100 \%)$ & & \\
II & 5 & $4(80 \%)$ & 1 & \\
III & 1 & & & 1 \\
IV & 19 & $4(21,1 \%)$ & 2 & 13 \\
IPI ajustado a edad & & & & \\
O (riesgo bajo) & 5 & $5(100 \%)$ & & \\
1 (riesgo intermedio) & 4 & $3(75 \%)$ & 1 & \\
2-3 (riesgo alto) & 15 & $2(13,3 \%)$ & 1 & 12 \\
Sin dato IPI & 6 & $3(50 \%)$ & & 3 \\
\hline
\end{tabular}

RC: remisión completa. RP remisión parcial. LDCG: linfoma difuso de células grandes.

La mediana de sobrevida para el grupo con LNH fue de 9 meses, con sobrevida de sólo 45,5\% a 12 meses de diagnosticado el linfoma, destacando una alta mortalidad en el primer año. La pendiente en la curva de mortalidad disminuye al avanzar el tiempo, volviendo a presentar descensos significativos a los 24 y 48 meses, con tasas de sobre vida de 41,5 y $36 \%$, respectivamente.

Comparando el tipo histológico de LNH (LDCG vs linfoma de Burkitt) no hubo diferencia significativa en la sobrevida, pero nuevamente se observó una alta mortalidad en ambos grupos. La sobrevida a 12 meses fue de 43,2 y $63,6 \%$, respectivamente, para los tipos histológicos recién mencionados. La falta de significación estadística es posiblemente debido al tamaño de ambos grupos.

Por último, la sobrevida según IPIae tuvo una clara diferencia a favor del grupo con IPIae más bajo. La sobrevida a un año del grupo IPIae $0-1$ fue de 84,3 vs $13,2 \%$ en el grupo IPIae $2-3$

\section{Conclusiones}

En el país, la mediana de edad para todos los pacientes con LNH, independiente de su condición de infección por VIH, es de 54 años, y para LH es de 37 años ${ }^{20}$. La comparación con nuestra casuística revela una edad similar para pacientes con LH. La mediana de edad para los casos de LNH fue de 42 años, edad inferior comparada con la de

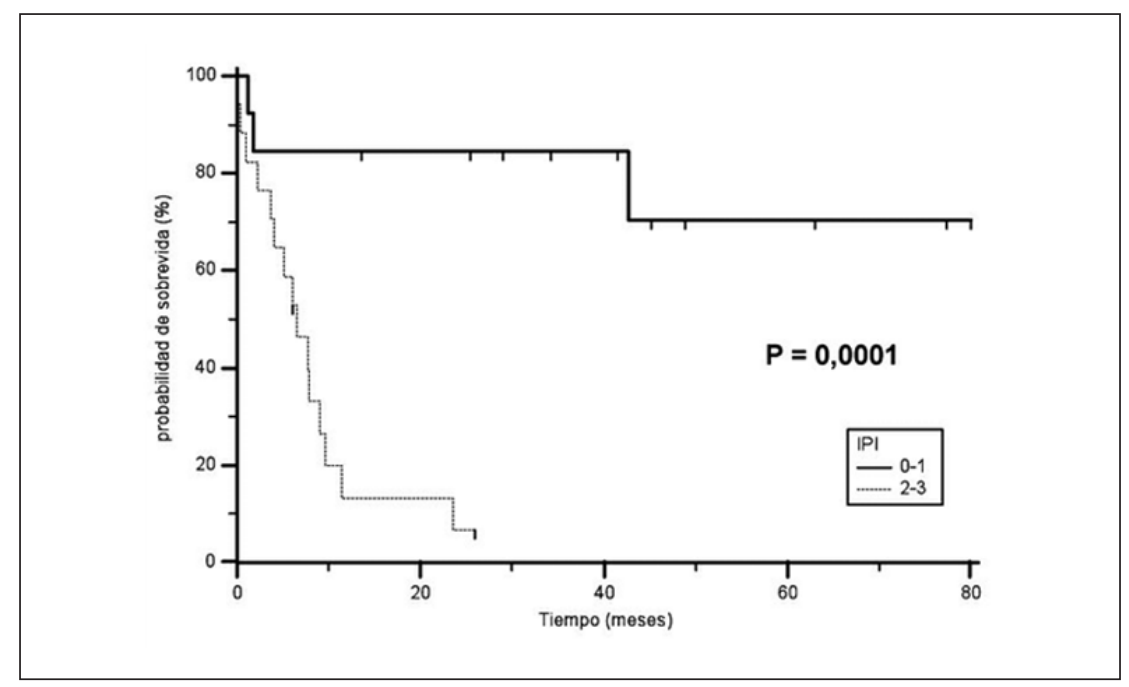

Figura 1. Análisis de sobrevida, según socre IPI curva Kaplan Meier para linfomas no Hodgkin. Hospital Clínico San Borja-Arriarán/Fundación Arriarán 2011-2008.

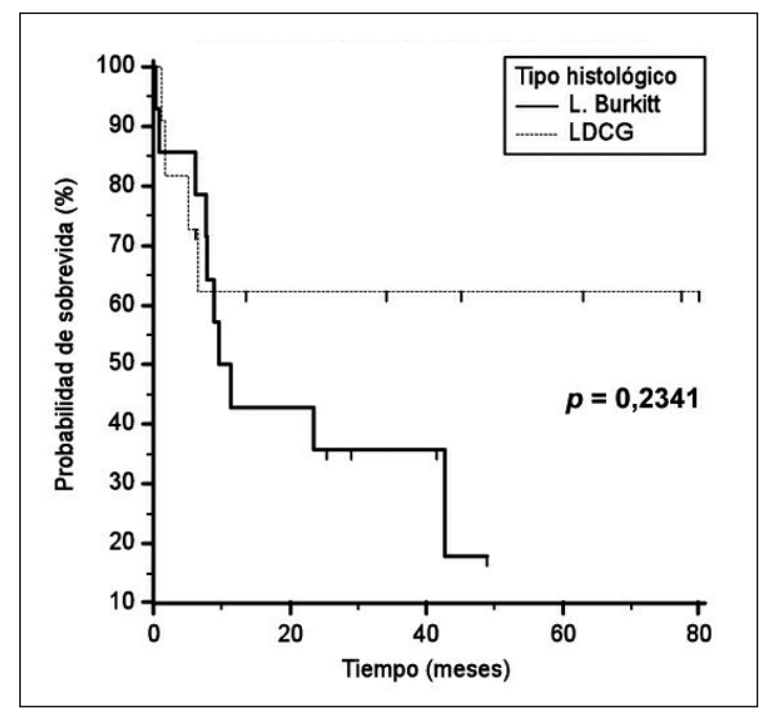

la población general, probablemente debido a la menor edad de los pacientes con VIH. Existe un predominio de pacientes del sexo masculino con una relación hombre/ mujer de 14:1 comparado con la población general de 1,5: 1, explicado por la mayor frecuencia de hombres en la población infectada por VIH en Chile. A nivel internacional la mediana de edad para pacientes con LNH relacionado a infección por $\mathrm{VIH}$ ha sido de 39 años, con $76 \%$ de sexo masculino ${ }^{21}$, en forma similar a lo observado en nuestro estudio.

Los tipos histológicos descritos como más frecuentes en linfomas relacionados a SIDA en la literatura científica corresponden a LDCGB y linfoma de Burkitt ${ }^{22}$ lo que se observó también en nuestra casuística. Hubo un caso ais-
Figura 2. Análisis de sobrevida-curva Kaplan Meier para linfomas no Hodgkin. Hospital Clínico San BorjaArriarán/Fundación Arriarán 2011-2008. 
lado de subtipo linfoma inmunoblástico concordante con la disminución de este tipo de linfoma desde la aparición de TARV altamente activa. La ausencia de pacientes con diagnóstico de LNH primario del sistema nervioso central y LNH de cavidades no es explicable sólo por la disminución de su frecuencia observada con el uso de TARV y extensamente descrita ${ }^{23,24}$ sino que por diversas razones que van probablemente desde falta de registro a dificultades técnicas de realizar biopsias estereotáxicas cerebrales y la agresividad de este tipo de linfomas con una muerte precoz, análisis que requiere mayor estudio y que se escapa de los objetivos de este trabajo.

La mayoría de los pacientes presentaron un estadio avanzado de la enfermedad según la clasificación de Ann Arbor, ya fuese estadio III con compromiso ganglionar supra e infradiafragmático o estadio IV que implica un compromiso extranodal, concordante con la agresividad de esta neoplasia en este grupo de pacientes. En casi $40 \%$ de los pacientes se realizó el diagnóstico de infección por VIH durante el proceso diagnóstico y de etapificación del linfoma, por lo tanto, la sospecha y luego confirmación diagnóstica de su condición de infectado fue debido al debut de su patología tumoral. Esto puede reflejar un tardío acceso al diagnóstico de la infección por VIH, el cual está limitado por las regulaciones nacionales actuales que requieren de una extensa y engorrosa consejería para la toma de la muestra y su posterior notificación.

Los pacientes con LNH recibieron una primera línea de quimioterapia (CHOP) en su gran mayoría (26 de 30 pacientes) con dosis total y en algunos casos con dosis reducida; el 73\% logró completar 5 a 6 ciclos de quimioterapia CHOP lo que indica una buena tolerancia a este tipo de tratamiento.

Sólo a algunos de los pacientes (4/15) que progresaron post quimioterapia CHOP se les indicó algún tipo de quimioterapia de rescate con malos resultados, debido a intolerancia, aplasia medular, sepsis y progresión de la enfermedad, lo que determinó la muerte en la totalidad de los casos.

La mayoría de los pacientes con linfoma de Burkitt no recibió en su inicio la terapia específica para este tipo de linfoma considerando su condición basal y el alto riesgo de citopenias prolongadas posterior a estos ciclos de quimioterapia. Se les ofreció una quimioterapia estándar con CHOP, progresando la enfermedad en 10 de 14 pacientes. Los pacientes con linfoma de Burkitt que progresaron, fallecieron pese a recibir posteriormente otro tipo de quimioterapia (tres pacientes). Los resultados muestran que la terapia CHOP en los casos de linfoma de Burkitt es insuficiente para controlar la enfermedad debiendo evaluarse los casos que puedan recibir quimioterapia específica para este tipo histológico en primera línea con el objetivo de mejorar la sobrevida global en este subgrupo de pacientes.
Los cuatro pacientes con linfoma de Burkitt que permanecían vivos hasta el último control fueron aquellos con estadio localizado y con IPI de 0 y 1 punto (bajo riesgo).

En relación a la búsqueda de factores pronósticos en las fichas clínicas se encontró con la dificultad de la inexistencia de evaluación de la escala de estado funcional de los pacientes, lo que obligó en algunos casos a inferir su condición por la evolución clínica y en $6(20 \%)$ de los pacientes no se realizó el examen LDH lo que imposibilitó asignarle un valor de la escala internacional pronóstica. Es necesario reforzar la educación y vigilancia de los equipos hematológicos para que los médicos tratantes de los pacientes con linfoma, independiente de su condición de VIH, apliquen el índice pronóstico internacional que incluye la etapificación, la escala de estado funcional y el valor de LDH. En los casos de LH se consigna el curso agresivo de la enfermedad; sin embargo, el escaso número de pacientes impide sacar mayores conclusiones.

El LNH en los pacientes infectados por VIH persiste en nuestro medio como una enfermedad de pésimo pronóstico, con frecuente progresión y recaídas de su enfermedad $\mathrm{y}$, adicionalmente, muertes provocadas por aplasia medular post quimioterapia y sus complicaciones infecciosas. En el concierto internacional, los estudios revelan en los últimos años una mejoría en el pronóstico de esta enfermedad, siendo similar a los pacientes sin infección por VIH, apoyando el uso de esquemas terapéuticos intensivos que incluyan rituximab ${ }^{25}$, el que no está disponible en nuestro medio según las guías PANDA. Actualmente la mayoría de los pacientes con linfoma e infección por VIH logran una curación de la enfermedad con sobrevida global a 5 años mayor de $60 \%{ }^{26}$, situación que no se observa en la casuística nacional. La incorporación de rituximab y la aplicación de esquemas de quimioterapia ajustados a la histología y factores de riesgo en el contexto de una mejoría general de la atención de salud de los pacientes del sistema público deberían, en el futuro, mejorar nuestros resultados locales.

El índice internacional pronóstico sigue siendo una herramienta útil para establecer la sobrevida de los pacientes con LNH, independiente de su condición de infección por VIH. Al analizar nuestros pacientes se consignan grupos de mejor pronóstico y sobrevida global como aquellos con LDCG con estadio localizado y con IPI bajo y grupos de pésimo pronóstico como aquellos con linfoma de Burkitt, LNH de estirpe T y linfoma inmunoblástico con estadios avanzados (III y IV) y con IPI alto (2-3) en que, pese a la terapia ofrecida, la sobrevida fue de pocos meses, por lo que se debe seguir estudiando nuevas y mejores alternativas terapéuticas para este grupo de pacientes. La consideración de estos factores pronósticos puede servir de orientación para ofrecer una terapia ajustada a su riesgo y no protocolos de quimioterapia uniforme para todos los pacientes. 


\section{Resumen}

Introducción: Los linfomas de Hodgkin (LH) y no Hodgkin (LNH) se asocian con alta frecuencia al síndrome de inmunodeficiencia humana en adultos. Objetivo: Sistematizar los aspectos clínicos e histológicos de los linfoma que afectan a pacientes con SIDA, su tratamiento y resultados globales en nuestra institución. Pacientes y Métodos: Análisis retrospectivo de pacientes con linfoma asociado a VIH entre enero de 2001 y diciembre de 2008 en el complejo hospitalario San Borja Arriarán. Resultados: Se obtuvo información de 30 pacientes con LNH y $7 \mathrm{LH}$, con una mediana de 40 años. Los tipos histológicos predominantes fueron linfoma de Burkitt (47\%), linfoma difuso de células grandes de estirpe B (37\%) y LNH de estirpe T (10\%). No se diagnosticaron LNH del SNC ni linfoma de cavidades. Casi la totalidad de los pacientes $(86,7 \%)$ con LNH se trataron con esquema CHOP, 57\% de quienes recibieron tratamiento presentaron progresión o recaída desde remisión completa, ofreciéndoles una quimioterapia de rescate a cuatro pacientes. El 73\% de los pacientes que recibieron CHOP lograron completar entre cinco y seis ciclos de quimioterapia. El uso de quimioterapia $\mathrm{CHOP}$ para el subgrupo de pacientes con linfoma de Burkitt alcanzó bajos porcentajes de remisión completa y mayoritariamente progresó la enfermedad, siendo esta quimioterapia, inefectiva para mejorar la sobrevida, especialmente en los pacientes de riesgo alto. Se encontraron diferencias estadísticamente significativas en sobrevida según el IPIae (índice internacional pronóstico ajustado por edad) al ingreso. Conclusión: El LNH en los pacientes con VIH tratados con los protocolos de quimioterapia PANDA persiste en nuestro medio como una enfermedad de muy mal pronóstico comparado con los resultados en la literatura internacional. La incorporación de nuevos fármacos de demostrada utilidad como rituximab y esquemas específicos de quimioterapia podrían mejorar estos resultados. El establecimiento de grupos pronósticos establecidos por IPIae puede orientar el trabajo clínico para el uso de quimioterapia ajustada a su riesgo específico y optimizado según tipo histológico.

\section{Referencias bibliográficas}

1.- Herida M, Mary-Krause M, Kaphan R, Cadranel J, Poizot-Martin I, Rabaud C, et al. Incidence of non AIDS-defining cancers before and during the highly active antiretroviral therapy era in a cohort of human immunodeficiency virus infected patients. J Clin Oncol. 2003; 21: 3447-53.

2.- Goedert J J, Coté T R, Virgo P, Scoppa S M, Kingma D W, Gail M H, et al. Spectrum of AIDS-associated malignant disorders. Lancet 1998; 351: 1833-9

3.- Biggar R J, Jaffe E S, Goedert J J, Chaturvedi A, Pfeiffer R, Engels E A. Hodgkin lymphoma and immunodeficiency in persons with HIV/AIDS. Blood 2006; 108: 3786-91.

4.- Jaffe E S, Harris N L, Stein H, Vardiman J W, eds. World Health Organization classification of tumours: pathology and genetics of tumours of haematopoietic and lymphoid tissues. Lyon, France: IARC Press; 2001: 237-52

5 Mounier N, Spina M, Gisselbrecht C. Modern management of non-Hodgkin lymphoma in HIV-infected patients. Br J Haematol 2006; 136: 685-98.

6.- Mocroft A, Katlama C, Johnson AM, Pradier C, Antunes F, Mulcahy F, et al. AIDS across Europe, 1994-98: the EuroSIDA study. Lancet 2000; 356: 291-6.

7.- International Collaboration on HIV and Cancer Highly active antiretroviral therapy and incidence of cancer in human immunodeficiency virus-infected adults. J Nat Cancer Inst 2000; 92: 1823-30.
8.- Ratner L, Lee J, Tang S, Redden D, Hamzeh F, Herndier B, et al. Chemotherapy for human immunodeficiency virus-associated non-Hodgkin's lymphoma in combination with highly active antiretroviral therapy. J Clin Oncol 2001; 19(8): 2171-8.

9.- Departamento de Epidemiología, Ministerio de Salud, Chile. Evolución del SIDA en Chile 1994-2008.

10.- Wolff M R, Northland R A, Segovia J P, Beltrán C B, Valdés M M, Albornoz M D, et al. Características clínicas e historia natural de la infección por virus de la inmunodeficiencia humana. Estudio en una población chilena atendida en un centro piloto multiprofesional. Rev Med Chile 1995; 123: 61-73.

11.- Wolff M R, Diomedi A A, Morales O B, Bidart T H, Dabanch J P, Bustamante C M, et al. Seguimiento prospectivo de una población infectada por VIH con y sin posibilidades de terapia anti-retroviral: impacto en sobrevida y complicaciones. Rev Med Chile 2001; 129: 886-94.

12.- Boletín epidemiológico CONASIDA MINSAL Chile, 2004.

13.- Osorio G, Montenegro C. Linfomas asociados a infección por virus de inmunodeficiencia humana en un complejo hospitalario de la Región Metropolitana, Chile: 1990-2002. Reporte de 14 casos y revisión. Rev Chilena Infectol 2007; 24 (2): 117-24.

14.- Carbone P P, Kaplan H S, Musshoff K, Smithers D W, Tubiana M. Report of the Committee on Hodgkin's disease staging classification. Cancer Res 1971; 31: 1860-1.
15.- Cheson B D, Pfistner B, Juweid M E, Gascoyne R D, Specht L, Horning SJ, et al for the International Harmonization Project on Lymphoma. Revised response criteria for malignant lymphoma J Clin Oncol 2007; 25 : 579-86.

16.- The International Non-Hodgkin's Lymphoma Prognostic Factors Project: A predictive model for aggressive non-Hodgkin's lymphoma. N Engl J Med 1993; 329: 987-94.

17.- Hasenclever D, Diehl V. Prognostic score for advanced Hodgkin's disease. N Engl J Med 1998; 339: 1506-14.

18.- Sehn LH, Berry B, Chhanabhai M, Fitzgerald C, Gill K, Hoskins P, et al. The Revised International Prognostic Index (R-IPI) is a better predictor of outcome than the Standard IPI for patients with DLBCL treated with R-CHOP. Blood 2007; 109: 1857-61.

19.- James O, Armitage M D. Early-stage Hodgkin's lymphoma. N Engl J Med 2010; 363: 653-62.

20.- Protocolo Linfoma de Hodgkin y Linfoma no Hodgkin 2008 PANDA MINSAL Chile.

21.- Miralles P, Berenguer J, Ribera J M, Rubio R, Mahillo B, Téllez M J, et al on behalf of the Grupo de Estudio del SIDA Register of Systemic AIDS-Related Lymphomas. Prognosis of AIDS-related systemic non-Hodgkin lymphoma treated with chemotherapy and highly active antiretroviral therapy depends exclusively on tumor-related factors. J Acquir Immune Defic Syndr 2007; 44: 167-73.

22.- Levine A M, Seneviratne L, Espina B M, 
Wohl AR, Tulpule A, Nathwani BN, et al. Evolving characteristics of AIDS related lymphoma. Blood 2000; 96: 4084-90.

23.- Simard E P, Pfeiffer R M, Engels E A. Cumulative incidence of cancer among individuals with acquired immunodeficiency syndrome in the United States. Cancer 2011: 1; 117 (5): 1089-96.
24.- Vishnu P, Aboulafia D M. AIDS-related non-Hodgkin's lymphoma in the era of highly active antiretroviral therapy. Adv Hematol. 2012: 485943. doi: 10.1155/2012/485943. Epub 2012 Feb 6.

25.- Kieron Dunleavy, Wyndham H. Wilson How I treat HIV-associated lymphoma Blood 2012; 119: 3245-55
26.- Dunleavy K, Little RF, Pittaluga $S$, Grant N, Wayne A S, Carrasquillo J A, et al. The role of tumor histogenesis FDG-PET, and shortcourse EPOCH with dose-dense rituximab (SCEPOCH- RR) in HIV-associated diffuse large B-cell lymphoma. Blood 2010; 115 (15): 301724. 